PROCEEDINGS OF THE

AMERICAN MATHEMATICAL SOCIETY

Volume 125, Number 4, April 1997, Pages 1221-1228

S 0002-9939(97)03600-9

\title{
THE HAUSDORFF DIMENSION OF THE SMALE-WILLIAMS SOLENOID WITH DIFFERENT CONTRACTION COEFFICIENTS
}

\author{
KÁROLY SIMON \\ (Communicated by Mary Rees)
}

\begin{abstract}
In this paper we prove that the Hausdorff dimension of the SmaleWilliams solenoid $\bar{\Lambda}$ with different contraction coefficients $\lambda, \mu$ is given by the formula $\operatorname{dim}_{H}(\bar{\Lambda})=1+\frac{\log 2}{\log (1 / \max (\lambda, \mu))}$. Further, for $\lambda, \mu<\frac{1}{8}$ we prove that the Hausdorff dimension of each angular section is equal to $\frac{\log 2}{\log (1 / \max (\lambda, \mu))}$.
\end{abstract}

\section{INTRODUCTION}

In this paper we compute the Hausdorff dimension of the Smale-Williams solenoid $\bar{\Lambda}$ with different contraction coefficients $\lambda, \mu$. Let $P$ be the solid torus; i.e. $P=$ $S^{1} \times D$ where $D$ is the unit disk in $\mathbf{R}^{2}$. We define the map $f: P \rightarrow P$ by

$$
f(\theta, r, s)=(2 \theta(\bmod 1), \lambda r+\varepsilon \cos (2 \pi \theta), \mu s+\varepsilon \sin (2 \pi \theta)),
$$

where $0<\varepsilon, \mu, \lambda<1$ are constants. The image $f(P)$ is contained in $P$ and wraps twice around $P$. See $[\mathrm{Shu}]$ for more details and pictures. Throughout this paper we assume that $f$ is a one-to-one map. This implies that $\lambda, \mu<\frac{1}{2}$. We call the attractor $\bar{\Lambda}=\bigcap_{n=0}^{\infty} f^{n}(P)$ a Smale-Williams solenoid with different contraction coefficients. Y. Pesin and H. Weiss $[\mathrm{PeW}]$ gave a lower and upper bound for $\operatorname{dim}_{H} \bar{\Lambda}$. In Theorem 1 we prove that $\operatorname{dim}_{H} \bar{\Lambda}$ is just the upper bound given in $[\mathrm{PeW}]$. The idea of the proof is as follows: using a suitable projection we trace back the computation of the Hausdorff dimension of $\operatorname{dim}_{H} \bar{\Lambda} \subset \mathbf{R}^{3}$ to the computation of the Hausdorff dimension of another attractor $\Lambda \subset \mathbf{R}^{2}$. The difficulty is that $\Lambda$ is an attractor of a non-invertible map, so its unstable fibers intersect each other. Our main result is that we can apply a previous theorem of the author [S, Theorem 1] to compute $\operatorname{dim}_{H} \bar{\Lambda}$.

The idea of tracing back the computation of the Hausdorff dimension of an attractor of an invertible map to the computation of the Hausdorff dimension of the attractor of a non-invertible map which lies in one dimension less, has appeared e.g. in $[\mathrm{B}]$ and $[\mathrm{PoW}]$.

Received by the editors June 23, 1994 and, in revised form, February 27, 1995 and July 26, 1995.

1991 Mathematics Subject Classification. Primary 58F12; Secondary 58F15.

The author was partially supported by grant F4411 from the OTKA Foundation.

(C)1997 American Mathematical Society 
In Theorem 2, we assume that $\lambda, \mu<\frac{1}{8}$. In this case, we compute the Hausdorff dimension of each angular section $\bar{\Lambda} \cap D_{\varphi}$, where $D_{\varphi}=\{(\theta, r, s): \theta=\varphi$ and $(r, s) \in D\}$ for any $\varphi \in S^{1}$.

Further, we show that the assumptions of Bothe's theorem [B, Theorem A] hold, which immediately implies that the Hausdorff dimension of all angular sections are $\log 2 / \log (1 / \max (\lambda, \mu))$. If $\max (\lambda, \mu) \geq \frac{1}{8}$, then the assumptions of [B, Theorem A] do not hold, and in this case we cannot compute the Hausdorff dimension of all angular sections.

\section{Results}

Principal Assumption. As we have already said, in what follows we always suppose that $f$ is a one-to-one map. This implies that

$$
\lambda, \mu<\varepsilon \quad \text { and } \quad \lambda, \mu<\frac{1}{2}
$$

hold. Further, without loss of generality we may assume that

$$
\mu \leq \lambda
$$

The main result of this paper is that the Hausdorff dimension of the SmaleWilliams solenoid with different contraction coefficients $\lambda, \mu$ is given by the following formula:

\section{Theorem 1.}

$$
\operatorname{dim}_{H}(\bar{\Lambda})=1+\frac{\log 2}{\log \frac{1}{\max (\lambda, \mu)}} .
$$

This formula does not give the Hausdorff dimension of all angular sections, but if $\lambda, \mu<\frac{1}{8}$, then we can apply the following theorem:

Theorem 2. If $\max (\lambda, \mu)<\frac{1}{8}$, then the Hausdorff dimension of all angular sections is

$$
\operatorname{dim}_{H}\left(\bar{\Lambda} \cap D_{\varphi}\right)=\frac{\log 2}{\log \frac{1}{\max (\lambda, \mu)}}
$$

\section{Notations}

We put $I=S^{1} \times[-1,1]$. Observe that the first two components of $f(\theta, r, s)$ depend only on $\theta, r$, and the third component does not depend on $r$. Thus the restriction of $f$ to $(\theta, r) \in I$ is a function $F: I \rightarrow I$ which is semiconjugate to $f$. More precisely, on putting

$$
F(\theta, r)=(2 \theta(\bmod 1), \lambda r+\varepsilon \cos (2 \pi \theta))
$$

we have

$$
F \circ \Pi=\Pi \circ f
$$

where $\Pi(\theta, r, s)=(\theta, r)$. It is important to note that $F$ is not a one-to-one map. One can easily see that the attractor $\Lambda=\bigcap_{n=0}^{\infty} F^{n}(I)$ is just the projection of $\bar{\Lambda}$ :

$$
\Lambda=\Pi(\bar{\Lambda}) \text {. }
$$


Further, $\Lambda$ consists of uncountably many smooth "wall-to-wall" curves in $I$. These curves are the graphs of the functions $\Lambda_{\left(i_{1}, i_{2}, \ldots\right)}: S^{1} \rightarrow[-1,1],\left(i_{1}, i_{2}, \ldots\right) \in$ $\{0,1\}^{\mathbf{N}}$, defined by

$$
\Lambda_{\left(i_{1}, i_{2}, \ldots\right)}(\varphi)=\varepsilon \sum_{i=1}^{\infty} \lambda^{k-1} \cos 2 \pi \frac{\varphi+m_{k}}{2^{k}},
$$

where $m_{i}=i_{1}+2 i_{2}+\cdots+2^{k-1} i_{k}$ for all $k \in \mathbf{N}, \varphi \in S^{1}$ and

$$
\Lambda=\bigcup\left\{\left(\varphi, \Lambda_{\left(i_{1}, i_{2}, \ldots\right)}(\varphi)\right): \varphi \in S^{1},\left(i_{1}, i_{2}, \ldots\right) \in\{0,1\}^{\mathbf{N}}\right\} .
$$

Sometimes we write $\Lambda_{\left(i_{1}, i_{2}, \ldots\right)}$ instead of $\left(\varphi, \Lambda_{\left(i_{1}, i_{2}, \ldots\right)}(\varphi)\right)$.

\section{Some lemmas And a Proposition}

To prove Theorem 1 (Theorem 2) we have to prove that the assumptions of $[\mathrm{S}$, Theorem 1] ([B, Theorem A $]$ ) hold respectively. Actually, what we need is a uniformly hyperbolic version of [S, Theorem 1] which is our Proposition below.

Those hypotheses of the Proposition and [B, Theorem A] which are hard to check in the present set-up are established in this section in Lemmas 1 and 2 which in turn require Facts 1 and 2.

Proposition 1. Let $A, B$ be intervals. Suppose that $F: A \times B \rightarrow A \times B, F(x, y)=$ $(h(x, y), g(y))$, satisfies the following conditions:

(i) there is a partition of $B$ into intervals $\Delta(1), \ldots, \Delta(l)$ such that $\left|g^{\prime}(y)\right| \geq c>1$ $(\forall y \in \Delta(i) 1 \leq i \leq l)$ and $g(\Delta(i))=B(1 \leq i \leq l)$;

(ii) $h_{x}^{\prime} \equiv \lambda$;

(iii) $0<\lambda<\min \left(\frac{1}{c}, \frac{1}{l}\right)$.

The attractor $\Lambda=\bigcap_{n=0}^{\infty} F^{n}(S)$ consists of smooth curves $\Lambda_{i_{1}, i_{2}, \ldots}$, where $1 \leq$ $i_{k} \leq l$ for all $k \in \mathbf{N}$ :

$$
\Lambda=\bigcup \Lambda_{\left(i_{1}, i_{2}, \ldots\right)}
$$

Moreover, if in addition:

(iv) the angle between any curve $\Lambda_{\left(i_{1}, i_{2}, \ldots\right)}$ and the stable direction is greater than a fixed constant, and

(v) any two curves $\Lambda_{\left(i_{1}, i_{2}, \ldots\right)}$ and $\Lambda_{\left(j_{1}, j_{2}, \ldots\right)}, i_{1} \neq j_{1}$, intersect each other in at most one point at which the angle between these curves is greater than a positive constant,

then

$$
\operatorname{dim}_{H}(\Lambda)=1+\frac{\log l}{\log \frac{1}{\lambda}}
$$

Proof. We start with the special case $A=B$ and show first that the assumptions [S, Theorem 1] are satisfied.

To check them one-by-one, I(a) and (b) immediately follow from (i) with $\lambda_{2}=\frac{1}{c}$ while I(c) follows from the definition of $h$ with $\lambda_{1}$ between $\lambda$ and $\min \left(\frac{1}{c}, \frac{1}{l}\right)$ ((iii) allows us to choose such a $\lambda_{1}$ ) whence II clearly holds.

III coincides with (v) above.

Next we show that the conclusion of $[\mathrm{S}$, Theorem 1] implies the conclusion of the Proposition. 
The conclusion of $[\mathrm{S}$, Theorem 1] states that

$$
\operatorname{dim}_{H}(\Lambda)=1+s,
$$

where $s$ is the solution of the pressure formula $P(s \cdot \psi)=0$, further $P$ is the topological pressure (see [R]) and $\psi=\log h_{x}^{\prime}$. However, $h_{x}^{\prime} \equiv \lambda$ immediately implies that $s=\frac{\log l}{\log (1 / \lambda)}$. This completes the proof when $A=B$.

Consider now the general case: $F: A \times B \rightarrow A \times B$. Choose $\tau(y)=u \cdot y+b$ so that $\tau(B)=A$ and we define $\tilde{g}(\tilde{y}):=\tau\left(g\left(\tau^{-1} \tilde{y}\right)\right)$ for $\tilde{y} \in \widetilde{A}$. Furthermore, put $\widetilde{F}: A \times A \rightarrow A \times A$ and

$$
\widetilde{F}(x, y)=\left(h\left(x, \tau^{-1} \tilde{y}\right), \tilde{g}(\tilde{y})\right)
$$

for $(x, \tilde{y}) \in A \times A$. Let

$$
\widetilde{\Lambda}=\bigcap_{n=0}^{\infty} \widetilde{F}^{n}(A \times A) .
$$

Now we can apply our argument to $\widetilde{F}$; we find that

$$
\operatorname{dim}_{H} \widetilde{\Lambda}=1+\frac{\log l}{\log \frac{1}{\lambda}} \text {. }
$$

Further,

$$
\Lambda=T(\widetilde{\Lambda})
$$

holds for the bi-Lipschitz map $T(x, \tilde{y})=\left(x, \tau^{-1}(\tilde{y})\right)$ since $F(T(x, \tilde{y}))=T(\widetilde{F}(x, \tilde{y}))$. Now (16) and (17) together imply that

$$
\operatorname{dim}_{H} \Lambda=1+\frac{\log l}{\log \frac{1}{\lambda}}
$$

This completes the proof of the Proposition.

In applying the Proposition to Theorem 1, the only difficulty is to check (v). Before we do this in Lemma 1, we need to establish two facts.

Fix $0<\theta<\frac{1}{2}$ so that

$$
\sin 2 \pi \frac{\theta}{2}=\frac{1}{3}
$$

Fact 1. If $0 \leq \varphi \leq \theta$, then

$$
\Lambda_{\left(0, i_{2}, i_{3}, \ldots\right)}(\varphi)>\Lambda_{\left(1, j_{2}, j_{3}, \ldots\right)}(\varphi),
$$

and if $1-\theta \leq \varphi<1$, then

$$
\Lambda_{\left(0, i_{2}, i_{3}, \ldots\right)}(\varphi)<\Lambda_{\left(1, j_{2}, j_{3}, \ldots\right)}(\varphi),
$$

Proof. We prove (20). The verification of (21) requires similar calculations. Thus assume $0 \leq \varphi \leq \theta$. From the definition of $\theta$ in (19) we have that

$$
\cos 2 \pi \frac{\varphi}{2} \geq \cos 2 \pi \frac{\theta}{2}=\frac{\sqrt{8}}{3} .
$$


Together with (9) this implies

$$
\begin{aligned}
\Lambda_{\left(0, i_{2}, i_{3}, \ldots\right)}(\varphi) & \geq \varepsilon\left(\cos \left(2 \pi \frac{\varphi}{2}\right)-\frac{\lambda}{1-\lambda}\right) \\
& \geq \varepsilon\left(\frac{\sqrt{8}}{3}-\frac{\lambda}{1-\lambda}\right)>\varepsilon(0.94-1)=-0.06 \varepsilon
\end{aligned}
$$

On the other hand, to estimate $\Lambda_{\left(1, j_{2}, j_{3}, \ldots\right)}(\varphi)$ we observe that in this case $m_{1}=1$ and thus $m_{2}=m_{1}+2 j_{2}$; i.e. $m_{2}=1$ or $m_{2}=3$.

If $m_{2}=1$, then the coefficient of $\lambda$ in (9) is

$$
\cos 2 \pi \frac{\varphi+m_{2}}{2^{2}}=-\sin 2 \pi \frac{\varphi}{2^{2}} .
$$

If $m_{2}=3$, then the coefficient of $\lambda$ in (9) is

$$
\cos 2 \pi \frac{\varphi+m_{2}}{2^{2}}=\sin 2 \pi \frac{\varphi}{2^{2}} .
$$

Thus in any case the absolute value of the coefficient of $\lambda$ in (9) is less than $\frac{1}{3}$ since

$$
\sin 2 \pi \frac{\varphi}{2^{2}}<\sin 2 \pi \frac{\theta}{2}=\frac{1}{3}
$$

holds. Now from (9) and (22) we get that

$$
\begin{aligned}
\Lambda_{\left(1, j_{2}, j_{3}, \ldots\right)}(\varphi) & <\varepsilon\left(\cos \left(2 \pi \frac{\varphi+1}{2}\right)+\frac{1}{3} \lambda+\lambda^{2}+\lambda^{3}+\lambda^{4}+\cdots\right) \\
& <\varepsilon\left(-\cos \left(2 \pi \frac{\varphi}{2}\right)+\frac{1}{6}+\frac{1}{2}\right) \leq \varepsilon\left(-\frac{\sqrt{8}}{3}+\frac{2}{3}\right)<-0.27 \varepsilon,
\end{aligned}
$$

whence, by (23) and (27)

$$
\Lambda_{\left(0, i_{2}, i_{3}, \ldots\right)}(\varphi)>-0.06 \varepsilon>-0.27 \varepsilon>\Lambda_{\left(1, j_{2}, j_{3}, \ldots\right)}(\varphi) .
$$

Fact 2. If $\theta \leq \varphi \leq 1-\theta$, then

$$
\begin{aligned}
\frac{d}{d \varphi} \Lambda\left(1, j_{2}, j_{3}, \ldots\right)(\varphi) & >-\frac{\varepsilon}{2}\left(\frac{\lambda}{2-\lambda}-\frac{1}{3}\right) \\
& >0>-\frac{\varepsilon}{2}\left(\frac{1}{3}-\frac{\lambda}{2-\lambda}\right)>\frac{d}{d \varphi} \Lambda_{\left(0, i_{2}, i_{3}, \ldots\right)}(\varphi) .
\end{aligned}
$$

Proof. From (9) it follows that

$$
\frac{d}{d \varphi} \Lambda_{\left(i_{1}, i_{2}, \ldots\right)}(\varphi)=-\frac{\varepsilon}{2} \sum_{k=1}^{\infty}\left(\frac{\lambda}{2}\right)^{(k-1)} \cdot \sin 2 \pi \frac{\varphi+m_{k}}{2^{k}}
$$

Suppose $m_{1}=0$. Then

$$
\begin{aligned}
\sum_{k=1}^{\infty}\left(\frac{\lambda}{2}\right)^{(k-1)} \cdot \sin 2 \pi \frac{\varphi+m_{k}}{2^{k}} & \geq \sin 2 \pi \frac{\varphi}{2}-\sum_{k=2}^{\infty}\left(\frac{\lambda}{2}\right)^{k-1} \\
& \geq \sin 2 \pi \frac{\theta}{2}-\frac{\lambda}{2-\lambda}=\frac{1}{3}-\frac{\lambda}{2-\lambda}>0
\end{aligned}
$$


since $\lambda$ is a fixed number less than $\frac{1}{2}$. Thus

$$
\begin{aligned}
\frac{d}{d \varphi} \Lambda_{\left(0, i_{2}, i_{3}, \ldots\right)}(\varphi) & =-\frac{\varepsilon}{2} \sum_{k=1}^{\infty}\left(\frac{\lambda}{2}\right)^{(k-1)} \cdot \sin 2 \pi \frac{\varphi+m_{k}}{2^{k}} \\
& <-\frac{\varepsilon}{2}\left(\frac{1}{3}-\frac{\lambda}{2-\lambda}\right)>0 .
\end{aligned}
$$

If $m_{1}=1$, then using a similar argument we find that

$$
\sum_{k=1}^{\infty}\left(\frac{\lambda}{2}\right)^{(k-1)} \cdot \sin 2 \pi \frac{\varphi+m_{k}}{2^{k}} \leq \frac{\lambda}{2-\lambda}-\frac{1}{3}<0 .
$$

Thus

$$
\frac{d}{d \varphi} \Lambda_{\left(1, j_{2}, j_{3}, \ldots\right)}(\varphi)>-\frac{\varepsilon}{2}\left(\frac{\lambda}{2-\lambda}-\frac{1}{3}\right)>0 .
$$

Now we are ready to prove our lemmas.

Lemma 1. The curves $\left(\varphi, \Lambda_{\left(0, i_{2}, i_{3}, \ldots\right)}(\varphi)\right)$ and $\left(\varphi, \Lambda_{\left(1, j_{2}, j_{3}, \ldots\right)}(\varphi)\right)$ intersect each other at exactly one point under an angle which is bounded away from zero by the uniform positive constant $2 \arctan \left(\frac{\varepsilon}{2}\left(\frac{1}{3}-\frac{\lambda}{2-\lambda}\right)\right)\left(\lambda<\frac{1}{2}\right.$ is fixed $)$.

Proof. It follows from Fact 1 that if $\varphi<\theta(\varphi>1-\theta)$, then $\Lambda_{\left(0, i_{2}, i_{3}, \ldots\right)}$ is above (below) $\Lambda_{\left(1, j_{2}, j_{3}, \ldots\right)}$. Thus $\left\{\varphi: \Lambda_{\left(0, i_{2}, i_{3}, \ldots\right)}(\varphi)=\Lambda_{\left(1, j_{2}, j_{3}, \ldots\right)}(\varphi)\right\} \subset(\theta, 1-\theta)$. On the other hand, it follows from Fact 2 that the function $\Lambda_{\left(0, i_{2}, i_{3}, \ldots\right)}(\varphi)\left(\Lambda_{\left(1, j_{2}, j_{3}, \ldots\right)}(\varphi)\right)$ is strictly decreasing (increasing) on the interval $(\theta, 1-\theta)$, thus there is exactly one $\varphi \in(\theta, 1-\theta)$ at which the curves $\Lambda_{\left(0, i_{2}, i_{3}, \ldots\right)}$ and $\Lambda_{\left(1, j_{2}, j_{3}, \ldots\right)}$ intersect each other. Further, it follows from Fact 2 that the angle between these curves at any $\varphi \in(\theta, 1-\theta)$ is at least $2 \arctan \left(\frac{\varepsilon}{2}\left(\frac{1}{3}-\frac{\lambda}{2-\lambda}\right)\right)$.

As a consequence of Lemma 1 we can now prove:

Lemma 2. If the distinct curves $\Lambda_{\left(i_{1}, i_{2}, \ldots\right)}$ and $\Lambda_{\left(j_{1}, j_{2}, \ldots\right)}$ intersect each other, then this intersection is transversal.

Proof. Suppose that $\left(i_{1}, i_{2}, \ldots\right)$ and $\left(j_{1}, j_{2}, \ldots\right)$ are distinct elements of $\{0,1\}^{\mathbf{N}}$. Further, assume that the curves $\Lambda_{\left(i_{1}, i_{2}, \ldots\right)}$ and $\Lambda_{\left(j_{1}, j_{2}, \ldots\right)}$ intersect each other under an angle $\beta$. What we want to prove is that

$$
\beta>0 .
$$

Let $k$ be the smallest number for which $i_{k} \neq j_{k}$.

If $k=1$, that is, $i_{1} \neq j_{1}$, then Lemma 1 applies. Assume that $k=2$. We define the curves $\gamma_{1}=F^{-(k-1)} \Lambda_{\left(i_{1}, i_{2}, \ldots\right)}$ and $\gamma_{2}=F^{-(k-1)} \Lambda_{\left(j_{1}, j_{2}, \ldots\right)}$, where $F^{-1}$ is taken according to $\left(i_{1}, \ldots, i_{k-1}\right)=\left(j_{1}, \ldots, j_{k-1}\right)$. One can easily see that $\gamma_{1} \subset$ $\Lambda_{\left(i_{k}, i_{k+1}, \ldots\right)}$ and $\gamma_{2} \subset \Lambda_{\left(j_{k}, j_{k+1}, \ldots\right)}$.

We can now apply Lemma 1 to conclude that the curves $\gamma_{1}$ and $\gamma_{2}$ intersect each other under an angle $\alpha>0$. Now apply $F^{k-1}$ to the curves $\gamma_{1}, \gamma_{2}$ to get back the curves $\Lambda_{\left(i_{1}, i_{2}, \ldots\right)}$ and $\Lambda_{\left(j_{1}, j_{2}, \ldots\right)}$. Using that the expansion (contraction) rate is 2 $(\lambda)$ in the unstable direction (stable direction), we obtain that $\tan \beta=\left(\frac{\lambda}{2}\right)^{k} \tan \alpha$, which proves (35). 


\section{Proofs}

Proof of Theorem 1. It is enough to show that

$$
\operatorname{dim}_{H} \Lambda=1+\frac{\log 2}{\log \frac{1}{\lambda}},
$$

since this and (8) imply that

$$
\operatorname{dim}_{H} \bar{\Lambda} \geq 1+\frac{\log 2}{\log \frac{1}{\lambda}}
$$

The reverse inequality is trivial (see [PeW, page 23]).

To prove (36), we apply the Proposition with the following substitution: $(x=$ $r, y=\theta), A=[-1,1], B=[0,1], h(r, \theta)=\lambda r+\varepsilon \cos \theta, g(\theta)=2 \theta(\bmod 1), l=2$ and $\Delta(1)=\left[0, \frac{1}{2}\right], \Delta(2)=\left[\frac{1}{2}, 1\right], c=2$. Now conditions (i)-(iii) of the Proposition obviously hold. Furthermore, it follows from (30) that the derivative of the function $\Lambda_{\left(i_{1}, i_{2}, \ldots\right)}(\varphi)$ is bounded above by a geometric series which is independent of $\left(i_{1}, i_{2}, \ldots\right)$. Thus we obtain that condition (iv) also holds, since the stable direction is the vertical one. Finally, Lemma 1 establishes condition (v). Thus we may apply the Proposition which establishes (36) and completes the proof of Theorem 1. (In (3) we have assumed that $\mu \leq \lambda$.)

To prove Theorem 2, we shall need to state a recent result due to H. G. Bothe which requires some further notation. Let

$$
\tilde{f}(t, x, y)=\left(\varphi(t), \lambda_{1}(t) \cdot x+z_{1}(t), \lambda_{2}(t) \cdot x+z_{2}(t)\right),
$$

where

$$
\varphi: S^{1} \rightarrow S^{1}, \quad \lambda_{1}, \lambda_{2}: S^{1} \rightarrow(0,1), \quad z_{1}, z_{2}: S^{1} \rightarrow(-1,1)
$$

are $\mathcal{C}^{\infty}$ mappings and $\frac{d \varphi}{d t}>1$. We define the projections

$$
\pi(t, x, y)=t, \quad \rho_{1}(t, x, y)=(t, x), \quad \rho_{2}(t, x, y)=(t, y) .
$$

We call $\widetilde{\Lambda}$ intrinsically inverse if for any arc $B \subset S^{1}$ and any two components $B_{1}, B_{2}$ of $\widetilde{\Lambda} \cap \pi^{-1}(B)$ the $\operatorname{arcs} \rho_{i}\left(B_{1}\right), \rho_{i}\left(B_{2}\right)$ are transverse in $S^{1} \times[-1,1]$ at each point of $\rho_{i}\left(B_{1}\right) \cap \rho_{i}\left(B_{2}\right)(i=1,2)$.

Theorem 3 (Bothe). Suppose that

(i) $\widetilde{\Lambda}$ is intrinsically transverse and

(ii) $\sup \lambda_{i}<\inf \dot{\varphi} \sup \dot{\varphi}^{-4 \log \inf \lambda_{i} / \log \sup \lambda_{i}}, i=1,2$.

Then

$$
\operatorname{dim}_{H}\left(\rho_{i}\left(\widetilde{\Lambda} \cap D_{t}\right)\right)=p_{i}
$$

holds for every $t \in S^{1}$, where $p_{i}$ is the unique number satisfying $P\left(p_{i} \log \lambda_{i}\right)=0(P$ is the topological pressure).

Now we are ready to prove our Theorem 2 .

Proof of Theorem 2. One can easily see that assumptions of Bothe's Theorem hold: (i) was stated as Lemma 2 , and it is immediate that (ii) holds if and only if $\lambda, \mu<\frac{1}{8}$. 
Thus Bothe's Theorem applies. Using that in this case $\lambda_{1}(t) \equiv \lambda, \lambda_{2}(t) \equiv \mu$, we have that $p_{1}=\frac{\log 2}{\log (1 / \lambda)}$ and $p_{2}=\frac{\log 2}{\log (1 / \mu)}$. Thus

$$
\operatorname{dim}_{H}\left(\bar{\Lambda} \cap D_{\varphi}\right) \geq \operatorname{dim}_{H}\left(\rho_{i}\left(\bar{\Lambda} \cap D_{\varphi}\right)\right) \geq \frac{\log 2}{\log \frac{1}{\max (\lambda, \mu)}}
$$

which completes the proof since the reverse inequality is trivial again.

\section{ACNOWLEDGEMENT}

The author would like to thank A. Manning and M. Pollicott for several useful discussions, and would also like to thank The Royal Society and Institute of Mathematics of the University of Warwick for their support and hospitality during the time this research was done.

\section{REFERENCES}

[B] H. G. Bothe, The Hausdorff dimension of certain attractors, Preprint 1993.

$[\mathrm{PeW}] \mathrm{Y}$. Pesin and H. Weiss, On the dimension of deterministic and random Cantor-like sets, symbolic dynamics, and the Eckmann-Ruelle Conjecture, Preprint 1994. MR 95k:28020

[PoW] M. Pollicott and H. Weiss, The dimension of some self affine limit sets in the plane and hyperbolic sets, Journal of Stat. Phys. 77 (1994), 841-866. MR 95h:58083

[Shu] M. Shub, Global stability of dynamical systems, Springer-Verlag, 1987. MR 87m:58086

[S] K. Simon, Hausdorff dimension for non-invertible maps, Ergod. Th. and Dyn. Systems 13 (1993), 199-212. MR 94c:58146

[R] D. Ruelle, Thermodynamic formalism, Reading Massachusetts, Encycl. of Math. and Appl. 5, 1978. MR 80g:82017

Institute of Mathematics, University of Miskolc, H-3515 Miskolc, Hungary 